\title{
Lifetimes and mean-free paths of hot electrons in the alkali metals
}

\author{
J. S. Dolado, ${ }^{1}$ V. M. Silkin, ${ }^{2}$ M. A. Cazalilla, ${ }^{3}$ A. Rubio, ${ }^{1,2,4}$ and P. M. Echenique ${ }^{1,2}$ \\ ${ }^{1}$ Materialen Fisika Saila, Kimika Fakultatea, Euskal Herriko Unibertsitatea, 1072 Posta kutxatila, \\ 20080 Donostia, Basque Country, Spain \\ and Centro Mixto CSIC-UPV/EHU, Donostia, Basque Country, Spain \\ ${ }^{2}$ Donostia International Physics Center (DIPC) \\ ${ }^{3}$ Department of Physics, Brown University, Providence, Rhode Island 02912-1843 \\ ${ }^{4}$ Laboratoire de Solides Irradiés (LSI), Ecole Polytechnique, Paliseau, France
}

(Received 13 March 2001; published 29 October 2001)

\begin{abstract}
We report first principle calculations of electron and hole inelastic lifetimes for alkali metals ( $\mathrm{Na}$ and $\mathrm{K}$ ) within the framework of the $G W$ approximation of many-body theory. The results show that at low energies the lifetimes are slightly smaller than prediction of a free-electron model, and that there is no noticeable momentum-direction dependence. We also look at the inelastic mean-free path $\lambda$, and conclude that the observed drops at a few $\mathrm{eV}$ in $\lambda$ for the alkali elements with a strong $d$-band character cannot be attributed to the effect of these $d$ bands in the lifetime, but to a crossover behavior of the velocity of the photoelectron.
\end{abstract}

DOI: 10.1103/PhysRevB.64.195128

PACS number(s): 73.20.-r, 71.10.-w

\section{INTRODUCTION}

One of the most important physical mechanisms for the decay of low-energy electronic excitations in metals is electron-electron interaction. Up to recent times, most of our knowledge on these matters relied on calculations of the electronic lifetimes in the free-electron gas (FEG) models, ${ }^{1}$ in which the modification of the electron states due to the lattice potential is not taken into account. The advent of a number of spectroscopic techniques such as time-resolved two-photon photoemission ${ }^{2}$ or the improved energy resolution of angle-resolved photoemission ${ }^{3}$ and scanning tunneling microscopy ${ }^{4}$ have renewed the interest in the particlehole dynamics of metals. On the theoretical side, the important development in computational possibilities, has allowed to carry out first principles calculations, mainly in the frame of the density-functional theory (DFT). ${ }^{5}$ These $a b$ initio calculations include the effect of the lattice potential, and therefore can be used as the starting point for a calculation of the lifetime within the framework of many-body perturbation theory. Here we have used the so-called $G W$ approximation ${ }^{6}$ to evaluate the lifetime of excited electrons in $\mathrm{Na}$ and $\mathrm{K}$. These systems are believed to be the prototypical examples of homogeneous electron gases. By this it is usually understood that the effects of the lattice potential can be neglected when calculating one-electron properties, and the only relevant parameter characterizing the system is the density of conduction electrons $n_{o}$, which is usually expressed in terms of the parameter $r_{s}=\left(3 / 4 \pi n_{0}\right)^{1 / 3}$, in units of the Bohr radius.

So far, calculations carried out in noble and simple metals ${ }^{7}$ within the $G W$ approximation and employing oneelectron orbitals obtained in the local-density approximation ${ }^{8}$ (LDA) have displayed important deviations from the predictions of the homogeneous electron gas due to band-structure effects. Taking into account the effect of the lattice potential changes both the magnitude and direction dependence of the lifetime of excited electrons and holes. Even a representative free-electron-like element, such as Al, exhibited a larger than expected sensitivity to crystal effects. ${ }^{7}$ In the present study, we have found that in the case of $\mathrm{Na}$ and $\mathrm{K}$ these changes are not so dramatic as, e.g., those found in Be. ${ }^{9}$ For Na and K, in the case of the lifetime, the anisotropy in the lifetime is approximately $25 \%$. Therefore, our results illustrate the extent to which the homogeneous electron gas provides a good description of these systems, as it is usually believed.

To the best of our knowledge, there are no available experimental data on the lifetime of excited electrons in $\mathrm{Na}$ and $\mathrm{K}$. Only measurements of the inelastic mean free path (IMFP) of excited electrons have been made in these systems. ${ }^{10}$ Motivated by these experiments, we also evaluate the IMFP as a function of the electron energy. We then discuss how our results can be compared to the experiments and, in particular, the adequacy or inadequacy of the usual definition of the inelastic mean free path, namely, as the lifetime times the group velocity. In this respect, we have found that one can account for the experimental observations if one assumes that the latter definition of IMFP applies only in the lowest-energy range whereas at higher energies the square root of the kinetic energy seems to provide a more suitable definition, as it has been found in previous studies. ${ }^{11}$

This paper is organized in the following way. In the next section, we give a brief account of the theory and approximations used in our analysis. Details of how the calculations have been actually carried out are also provided in that section. In Sec. III the results are presented. This section is divided in two subsections devoted to the description of the results for the electronic lifetime (Sec. III A) and the inelastic mean free path (Sec. III B) in $\mathrm{Na}$ and $\mathrm{K}$. The main conclusions of the present work have been summarized in Sec. IV. Finally we provide an appendix where we discuss how experiments extract the IMFP, and their inherent limits. Atomic units $\left(\hbar=e^{2}=m_{e}=1\right)$ are used in all mathematical expressions.

\section{THEORY, APPROXIMATIONS, AND CALCULATIONAL DETAILS}

The ground-state calculations were performed within the first principle total energy pseudopotential method ${ }^{12}$ of DFT 
in the Kohn-Sham formalism. We used the nonlocal norm conserving Troulliers- Martin pseudopotential. ${ }^{13}$ The selfconsistent Kohn-Sham equations have been calculated within a plane-wave formalism, in the LDA approach. ${ }^{14}$

The obtained LDA energies $\left(\epsilon_{\mathbf{k}, n}^{L D A}\right)$ and orbitals $\left(\phi_{n \mathbf{k}}\right)$ are a good starting point to calculate the self-energy $\Sigma(\mathbf{k}, \omega)$, and hence, the quasiparticle energies $\epsilon_{\mathbf{k}, n}^{Q P}$. These are obtained from the self-consistent equation

$$
\epsilon_{\mathbf{k}, n}^{Q P}=\epsilon_{\mathbf{k}, n}^{L D A}+\Delta \Sigma\left(\mathbf{k}, \epsilon_{\mathbf{k}, n}^{Q P}\right),
$$

where we define $\Delta \Sigma$ as $\Sigma-V_{x c}^{L D A}$, i.e., the self-energy minus the LDA exchange-correlation potential.

It is usual to expand the self-energy around the LDA energies and write

$$
\epsilon_{\mathbf{k}, n}^{Q P}=\epsilon_{\mathbf{k}, n}^{L D A}+Z_{\mathbf{k}, n} \Delta \Sigma\left(\mathbf{k}, \epsilon_{\mathbf{k}, n}^{L D A}\right),
$$

where $Z_{\mathbf{k}, n}=\left[1-\delta \Sigma(\mathbf{k}, \omega) / \delta \omega \|_{\epsilon_{\mathbf{k}, n}^{L D A}}\right]^{-1}$ is known as the renormalization constant. Taking the imaginary part in Eq. (2), we obtain the formula for the lifetime

$$
\tau_{\mathbf{k}, n}^{-1}=-2 Z_{\mathbf{k}, n} \operatorname{Im} \Sigma\left(\mathbf{k}, \epsilon_{\mathbf{k}, n}^{L D A}\right) .
$$

To calculate the self-energy we have followed the $G W$ approximation, ${ }^{6,15}$ and we have worked on the energy- shell (i.e., $Z_{\mathbf{k}, n} \equiv 1$ ). This approach has shown to provide a good description in other metallic systems ${ }^{7}$ and relies on the weakenergy dependence of the self-energy. In this frame the above equation can be written as: ${ }^{6,15}$

$$
\begin{aligned}
\tau_{n \mathbf{k}}^{-1}= & -2 \sum_{m} \int_{B Z(2 \pi)^{3}} \sum_{\mathbf{G}} \sum_{\mathbf{G}^{\prime}} B_{n m}^{*}(\mathbf{q}+\mathbf{G}) \\
& \times B_{n m}\left(\mathbf{q}+\mathbf{G}^{\prime}\right) \operatorname{Im} W_{\mathbf{G G}^{\prime}}(\mathbf{q}, \omega),
\end{aligned}
$$

with $\omega=\epsilon_{n \mathbf{k}}^{L D A}-\epsilon_{m \mathbf{k}-\mathbf{q}}^{L D A}$. Thus, in this framework, the lifetime is written in terms of the imaginary part screened coulomb potential $W$ (namely, proportional to $\operatorname{Im} \epsilon^{-1}$ ) and the matrix elements

$$
B_{n m}(\mathbf{q}+\mathbf{G})=\int d \mathbf{r} \phi_{n \mathbf{k}}^{*}(\mathbf{r}) e^{i(\mathbf{q}+\mathbf{G})} \phi_{m \mathbf{k}-\mathbf{q}}(\mathbf{r}),
$$

between the initial and final state. To account for the response of the system and compute the screened interaction, $W$, we have followed the so-called random-phase approximation (RPA). ${ }^{16,17}$

We refer the reader to Ref. 15 for details of the method and applications to simple and noble-metal electron/hole lifetimes.

\section{RESULTS}

\section{A. Lifetime}

In most experiments the lifetime are obtained resolved in energies and not in momentum. Therefore, in order to compare with experiments we need to perform an average. The simplest and most physical one is ${ }^{7}$ (a)

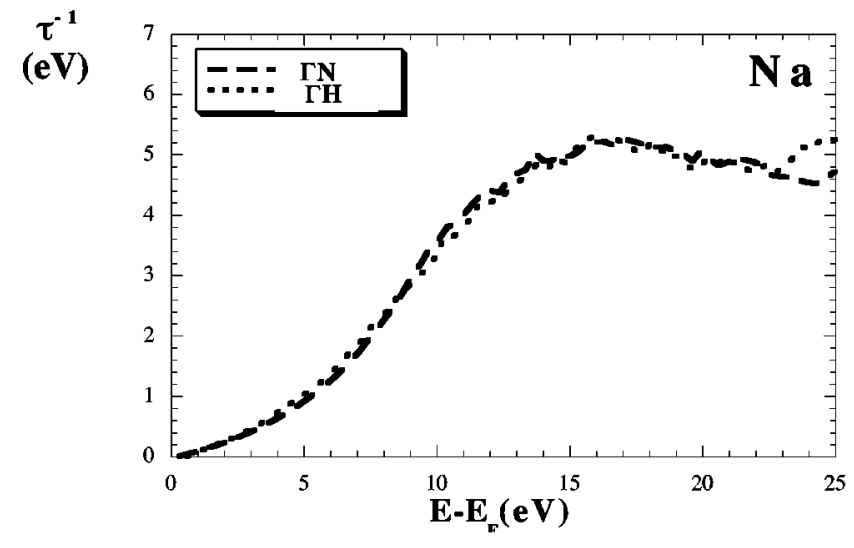

(b)

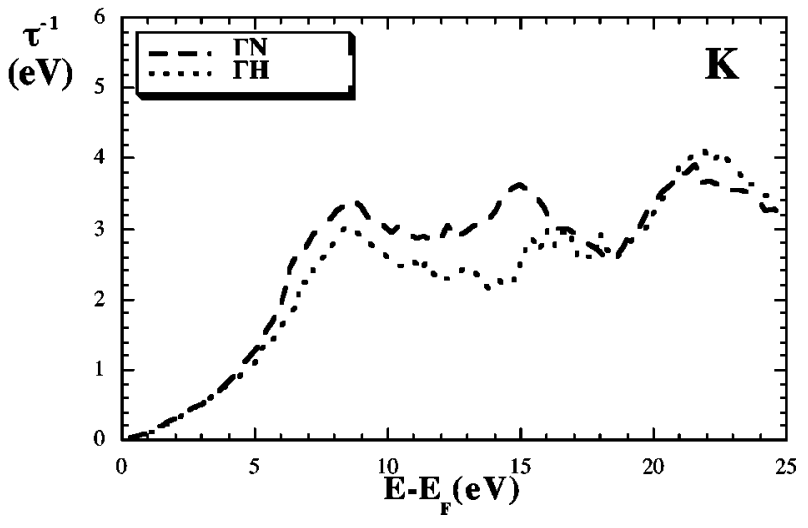

FIG. 1. (a) Linewidth for two different directions ( $\Gamma N$ and $\Gamma H$ ) for Na. Long-dashed lines stand for the $\Gamma N$ direction whereas shortdashed lines stand for the $\Gamma H$. (b) The same for $\mathrm{K}$.

$$
\tau_{\alpha}^{-1}(E)=\frac{\sum_{n} \int_{\mathcal{C}_{\alpha}} d \mathbf{k} \tau^{-1}(\mathbf{k}, n) \boldsymbol{\delta}(E-E(k, n))}{\sum_{n} \int_{\mathcal{C}_{\alpha}} d \mathbf{k} \delta(E-E(\mathbf{k}, n))} .
$$

where $\tau^{-1}(\mathbf{k}, n)$ is the inverse lifetime of a Bloch state $|\mathbf{k} n\rangle$, and $E(\mathbf{k}, n)$ its band energy. The averaged is performed over the Brioullin zone along a given crystallographic direction $\alpha{ }^{18}$ We find only a small dependence on the calculated lifetimes for $\mathrm{Na}$ when different directions are studied. This behavior can be observed in Fig. 1(a) where we plot the obtained results along two directions, $\Gamma N$ and $\Gamma H$. In Fig. 1(b) we show the same but for $\mathrm{K}$, where there is not an important difference (less than 10\%) up to $12 \mathrm{eV}$ above the Fermi level. Only in a small energy range around $15 \mathrm{eV}$ there is a noticeable directional dependence (always less than 30\% related to $d$ character of the bands). Thus, in this sense, alkali metals do not exhibit the important directional dependence found in elements such as $\mathrm{Be}, \mathrm{Cu}$, or $\mathrm{Au}$. This behavior could be expected since these systems are well described by the nearly free-electron model.

In Fig. 2(a) we show for Na the results of our calculations together with the FEG-RPA predictions. The dashed lines 


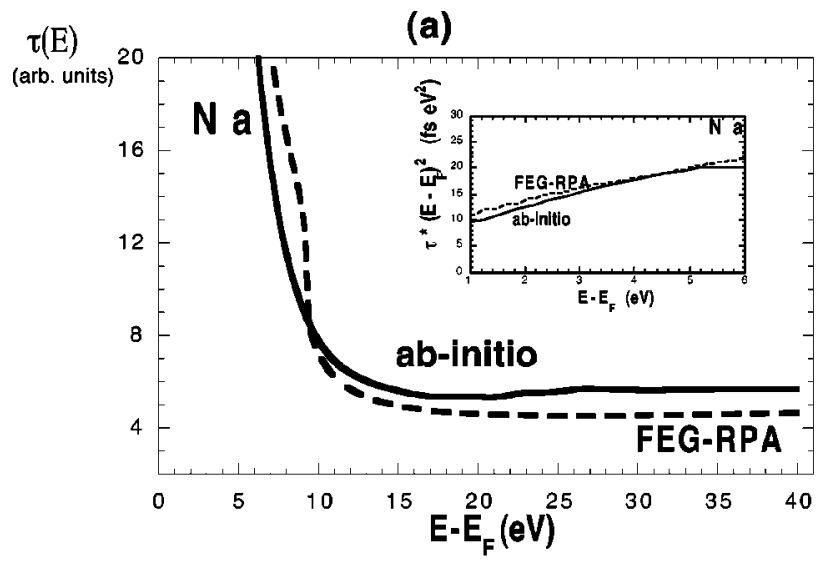

(b)

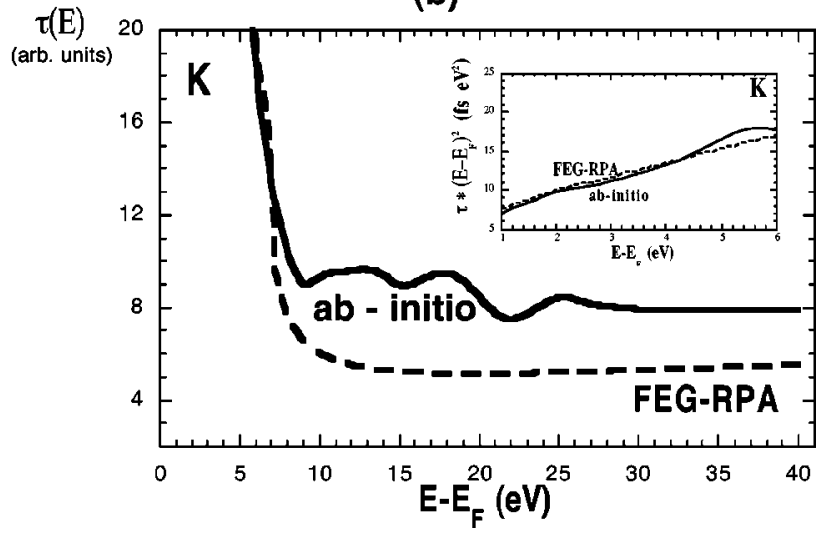

FIG. 2. (a) Hot-electron lifetime in Na compared with the FEG model. Inset: Averaged lifetime for $\mathrm{Na}$ in the low-energy regime, compared with the FEG model. (b) Hot-electron lifetime for $\mathrm{K}$ compared with a FEG model. Inset: Averaged lifetime for $\mathrm{K}$ in the low-energy regime compared with the FEG.

represent the FEG-RPA calculations, ${ }^{1}$ and our full-band calculation is denoted by the solid line. On average, the fullband model and the FEG give very similar lifetimes. This results were expected as long as the Fermi surface of $\mathrm{Na}$ (and of $\mathrm{K}$ ) is very much like a sphere. However, our results exhibit two clear regimes, similar to the ones observed in other simple metals as Aluminum ${ }^{7}$ : A low-energy regime (up to 10 eV) where the FEG overestimate the realistic lifetimes and a high-energy range (above $10 \mathrm{eV}$ ), where the FEG seems to underestimate the lifetimes. This last fact can be observed with more detail in the inset, where we have represented the averaged lifetime in the low-energy range. For $\mathrm{Al}$ the deviation is about $35 \%,{ }^{7}$ whereas in $\mathrm{Na}$ the maxima deviation (below $1 \mathrm{eV}$ ) is about $20 \%$.

In Fig. 2(b) we show our results for the K case. Potassium is a bit different because of empty $d$-bands above the Fermi level. We have now two competing effects: (i) increasing of the density of states (available phase space for decaying) that would reduce the lifetime, and (ii) overlap between the $d$ and sp-like band orbitals, that tend to increase the lifetime. ${ }^{19}$ As in the case of sodium, the low-energy range presents the same pattern, i.e., a deviation from the FEG, with slightly smaller lifetimes than those predicted from FEG. The low-

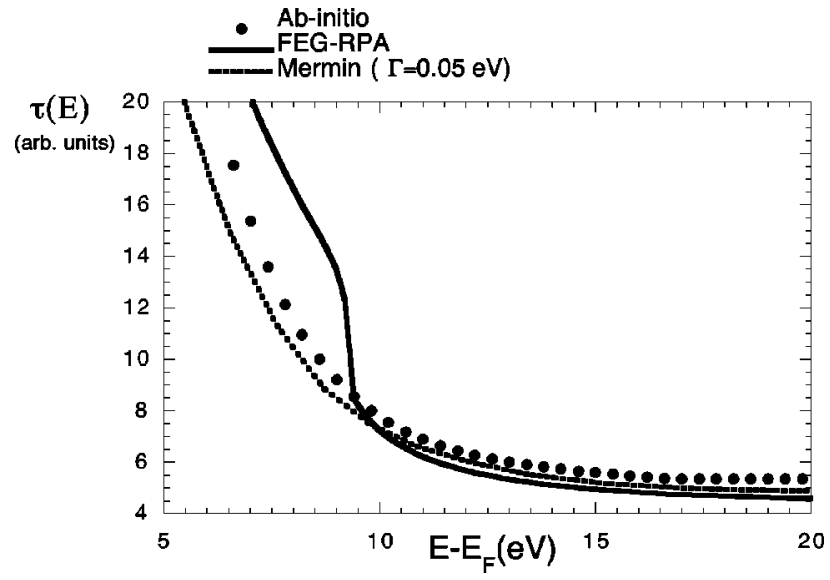

FIG. 3. Comparative study of the effect of a finite damping. With solid line we denote the results from a FEG calculation with a RPA response, whereas dashed lines stand for FEG with the Mermin's prescription.

energy regime has been explored in the inset, where we plot the average lifetime. In this case, the deviation with respect to the FEG behavior is smaller than in the Na case. Here the deviation is always lower than $15 \%$.

To study the effect of the plasmon width on the lifetime we have also computed the lifetimes with the Mermin's response function. ${ }^{20}$ In this procedure the dielectric function is

$$
\begin{aligned}
& \epsilon_{M e r \min }(\mathbf{q}, \omega) \\
& \quad=1+\frac{(1+i \gamma / \omega)\left[\epsilon_{L}(\mathbf{q}, \omega+i \gamma)\right]}{1+(i \gamma / \omega)\left[\epsilon_{L}(\mathbf{q}, \omega+i \gamma)-1\right] /\left[\epsilon_{L}(\mathbf{q}, 0)-1\right]},
\end{aligned}
$$

where $\epsilon_{L}$ is the Lindhard dielectric function ${ }^{16}$ and $\gamma$ a phenomenological damping (Fig. 3). The Mermin's response function is the simplest FEG response that incorporates a phenomenological damping to the Lindhard response function in a particle-conserving way, and allows to isolate easily the effect of the damping from other band structure effects. In Fig. 4 we show a comparison of FEG-RPA, FEG-Mermin and the $a b$ initio lifetimes. We see that effectively the lifetime obtained with the Mermin's prescription gives a more qualitative agreement with the ab initio calculation. In fact, it is also capable of reproducing the mentioned two regimes. Thus, although this pattern was thought as a band-structure effect, we have identified its origin in the plasmon width, being the effect of band-structure concentrated mainly in giving rise to a physical damping.

To complete this section devoted to the lifetimes we have investigated what happen with holes. Holes, in the FEG model are known to live shorter than their electron counterparts. ${ }^{15}$ Nevertheless, a recent first-principle calculation has shown that in noble metals $d$ holes exhibit longer inelastic lifetimes. ${ }^{7}$ Obviously noble metals are much more complex systems than alkalis, but the cited work illustrates the crucial importance of full-band calculations to describe correctly the hole dynamics. Na and $\mathrm{K}$ do not have occupied $d$ bands, so a priori one would expect that band structure 
(a)

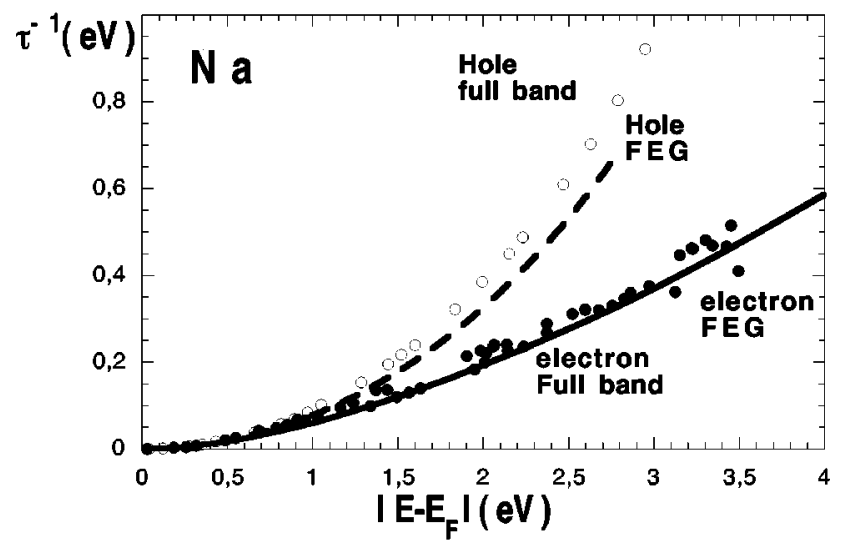

(b)

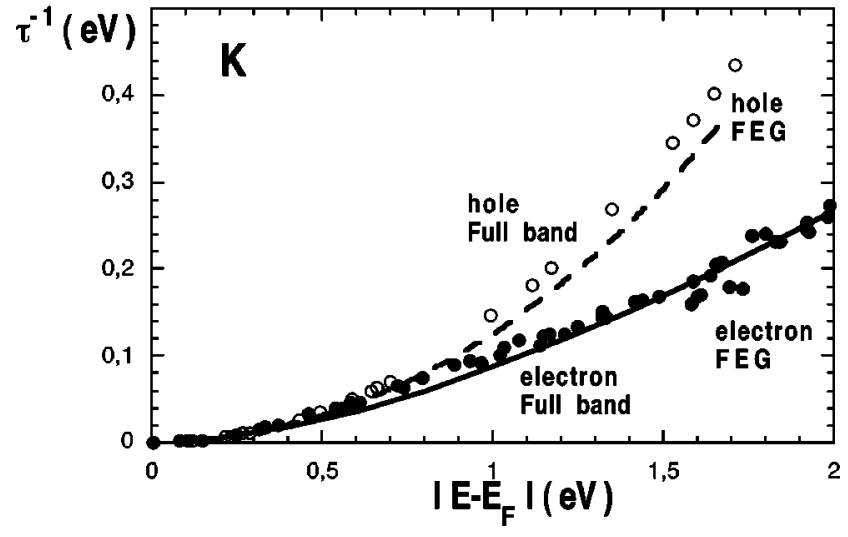

FIG. 4. (a) Electron and hole linewidths in $\mathrm{Na}$ and (b) for $\mathrm{K}$. Solid (open) circles denote the full-band electron (hole) linewidths, whereas solid (dashed) line represents the FEG-RPA model for electrons (holes).

were just a small correction (as in the case of electrons for small energies). Although our results seem to confirm this supposition, we find some differences. In Fig. 5(a) we have plotted our $a b$ initio lifetimes for electrons and holes (solid points), together the FEG-model results (solid lines). We find that, as founded for electrons, our $a b$ initio hole lifetimes are slightly shorter than those predicted within the FEG model. Another remarkable fact is that the deviation between full band and FEG lifetimes is now larger, showing that the crystal effects are even more important for holes than for electrons.

\section{B. Inelastic mean free path}

The inelastic mean free path is the average distance that an excited electron travels before inelasticaly decaying into another state. Namely, it is the product of the velocity of that give state per the lifetime $(\lambda=\tau v)$. Similarly to what was done for lifetimes, in order to make direct contact with experiment we have to perform an average. Thus, we have defined the IMFP as

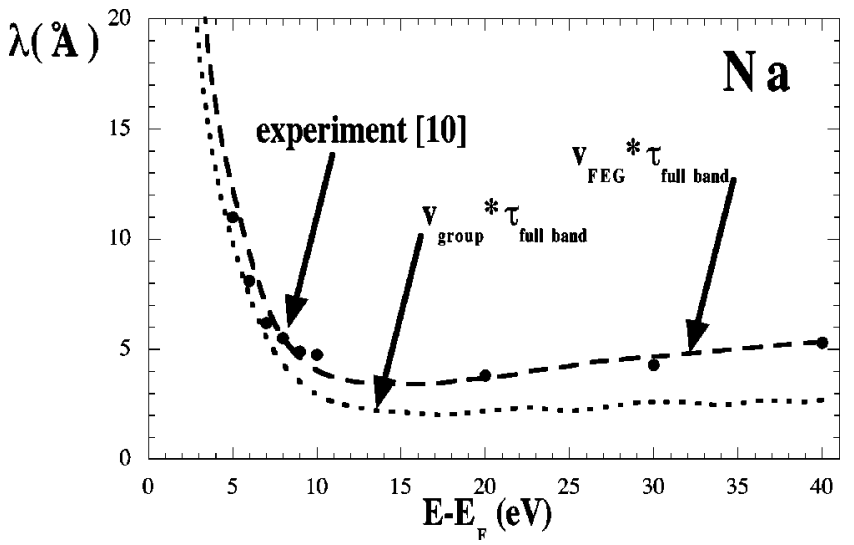

FIG. 5. IMFP for Na. Solid circles represent the experimental data. Dotted lines stand for the mean free path whereas the velocity we have employed the group velocity, and as effective lifetime, our full-band lifetime. With dashed lines we have represented the same as the dotted line, but changing the group velocity by the FEG velocity.

$$
\lambda(E)=\frac{\sum_{n} \int d \mathbf{k} \lambda(\mathbf{k}, n) \delta(E-E(\mathbf{k}, n))}{\sum_{n} \int d \mathbf{k} \delta(E-E(\mathbf{k}, n))},
$$

where $\lambda(\mathbf{k}, n)=v(\mathbf{k}, n) \tau(\mathbf{k}, n)$.

But, what velocity $v$ needs to be used here? A recent work $^{11}$ has remarked that the usual definition of the IMFP, namely, the lifetime times the group velocity fails when trying to compare with experiments, and employed as velocity the square root of the kinetic energy. In present work we have considered both possibilities, and we have seen that experiment seems to follow a crossover behavior, with a low-energy range where the usual group velocity seems to provide a good agreement with experiment, and a higherenergy range where it fails, and the free-electron velocities suits better. ${ }^{23}$

Few eV above the Fermi level the IMFP of heavy alkalis exhibit a dramatic reduction ("drops"). In the experiments the drops were attributed to the presence of unoccupied $d$ bands. However from our results we conclude that neither the IMFP calculated with the group velocity (dashed lines) nor calculated with the FEG velocity (dotted line) exhibit this behavior. We find that using the group velocity yield agood agreement at low energies $(\leqslant 10 \mathrm{eV})$, whereas at high energies $(\geqslant 20 \mathrm{eV})$ the IMFP seems to match the results obtained using the FEG velocity. Thus a possibility to reproduce the experimental IMFP would be to define a velocity depending on the energy (see Fig. 5):

$$
\begin{gathered}
v(E)=v_{\text {group }}(E) \quad E \simeq E_{F}, \\
v(E)=v_{F E G}(E) \quad E \gg E_{F},
\end{gathered}
$$

Thus, we consider the drop as a consecuence of a general mechanism: the crossover, i.e., the gradual change of the photoelectron velocity from the group velocity branch to the free-electron velocity. In this sense, heavy alkalies $(\mathrm{K}, \mathrm{Rb}$, 


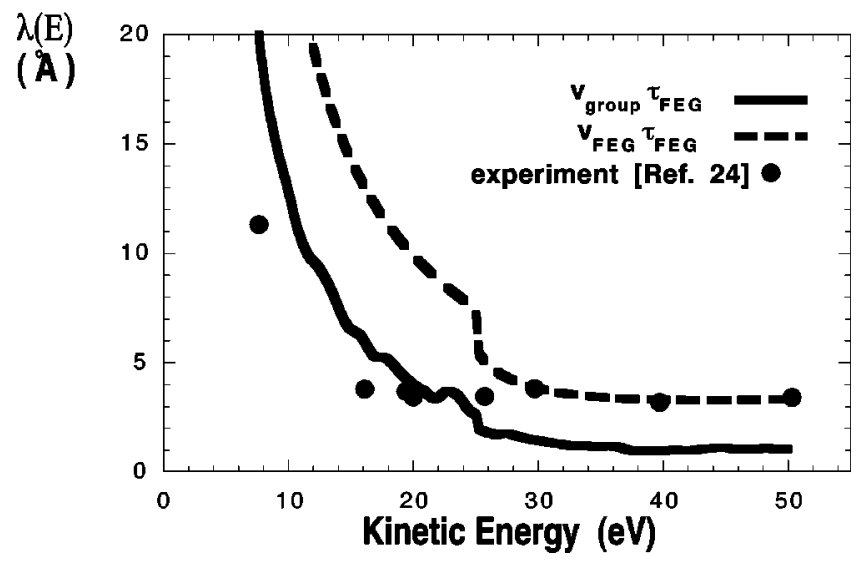

FIG. 6. IMFP for Si. The solid line represents the result obtained by employing as velocity the group velocity, whereas the dashed line uses FEG velocities. The experimental data from (Ref. 24) is represented by solid points. The group velocities have been calculated with a $10 \times 10 \times 10$ grid, 50 bands, and an energy cutoff of 40 Ry. The lifetimes have been calculated in a FEG model with an effective radius $r=2.0$ corresponding to a plasmon frequency $\omega_{p}$ $=16.6 \mathrm{eV}$.

Cs) are just a particular and relevant case. In those systems the $d$ bands give group velocities that differ enormously from the free-electron-like velocities, bringing out more abrupt crossovers, and finally drops. Independently of whether or not the measured IMFP have a drop there is a general pattern that can be only described by resorting to the mentioned crossover.

We observe that this crossover behavior for IMFP is a general experimental result, not linked to any particular system, and that it is an inherent mechanism of the photoemission phenomena. For example, experiments in silicon ${ }^{24}$ showed that at low energies the electronic inelastic mean free paths fall somewhat below the FEG behavior, showing the mentioned behavior. This fact is showed in Fig. 6, where the points stand for the experimental data and the lines (solid and dashed) are theoretical calculations . Another interesting element that exhibits the same trend is Beryllium. Johanson and Sernelius ${ }^{21}$ collected experimental data along two different crystallographic directions and performed a theoretical calculation within a FEG formalism. As in present work, they showed that experiment and theory agree fairly well at high energies, but the experiment values drop markedly below the FEG-RPA model for small energies. ${ }^{22}$ This conclusion is corroborated in a $a b$ initio calculation, ${ }^{9}$ where again the two-regime pattern is found for the IMFP.

An intuitive explanation for this crossover behavior could rely on the time that the photoelectron is in the solid. At large energies the photoelectrons leave the solid before feeling the lattice, and basically they behave as plane waves. And the opposite: At low energies the photoelectrons are into the solid time enough for seeing the lattice and becoming a Bloch state. We have here a technical comment: At large energies, in order to calculate the effective lifetimes we have employed our full-band formalism, i.e., we have used as wave functions the Bloch states, whereas if the photoelectron behaves as a free electron we should have employed as final wave function plane waves. We have checked that at large energies if the coefficients $\left|B_{i f}(\mathbf{q}+\mathbf{G})\right|^{2}$ are replaced by those obtained within a free-electron model, and we keep the full inclusion of the band structure in the evaluation of the response $\operatorname{Im}\left[-\epsilon_{\mathbf{G}, \mathbf{G}^{\prime}}^{-1}(\mathbf{q}, \omega)\right]$, the resultant lifetime coincides with that obtained with Bloch states as final states. Nevertheless the above explanation would mean to recognize that the IMFP calculation is inherently a surface problem, apart from the fact that neglects the main theoretical problem: only for states very closed to the Fermi level the quasiparticles are strictly well defined (linewidths much smaller than the energies), fact that prevents us from a direct one-electron analogy. However, if we still follow with the one electron point of view and put altogether energies and linewidths in the energy-band structure, instead of having lines, we would obtain noodlelike structures. Thus, the band structure would be masked by the intrinsic lifetimes. ${ }^{25}$ This effect has been alrady pointed by several authors ${ }^{3,26,27}$ as a important mechanism that leads to a distorsion of the bands and that the they look much more like free-electron bands.

\section{CONCLUSIONS}

We have calculated the lifetimes of $\mathrm{Na}$ and $\mathrm{K}$, in a fullband GW scheme, showing that (i) there is not such important anisotropy when studying different crystallographic directions as in other systems $(\mathrm{Cu}, \mathrm{Be} \ldots)$, (ii) at low energies we find, as in Ref. 7 a reduction for the lifetime compared to the FEG model, but with slight deviation (always lower than the $20 \%$ ), attributing this feature to the finite width of the plasmon. (iii) The $d$ bands of potassium make the lifetime bigger with respect to the FEG. (iv) With respect to the holes, we have seen that the inclusion of the full-band structure is even more important than in the case of electrons, showing a more noticeable reduction in the lifetime.

Furthermore we have provided a detailed study of the problems that one has to deal with when studying inelastic mean free paths. We have pointed out that not always experiment and theory have equal characteristic mean free paths, and when the characteristic mean free paths are of the order of the interatomic distance an special attention must be paid. Second, we have tried to explain the experimental data. When doing so, we have found a rich variety of problems, some of them already addressed in Ref. 11. Nevertheless, one aim of this paper is to suggest that the photoemission experiments might present two different regimes. So within this theoretical frame only the two asymptotic limits are accessibles. In any case, it is enough for explaining the pronounced drop in the IMFP of the heavier alkali metals. In Ref. 10, this behavior was attributed to electron-decay processes involving unoccupied $d$ bands. We have seen that this is not the case and that although the $d$ bands contribute to a little drop when the $d$ channel disappears, it is not enough to explain the experimental data. The existence of two regimes seems to be crucial to explain such a behavior. Furthermore, other different systems such as $\mathrm{Si}$ or Be, seem to exhibit the same features. This prediction needs to be checked in more systems, including transition metals and others. 


\section{ACKNOWLEDGMENTS}

We would like to thank I. Nagy for stimulating discussions. We also acknowledge support by the University of the Basque Country, and S.A. Iberdrola A.R. acknowledge support from the sabbatical program Salvador de Madariaga of the Spanish MEC (PR2000-0335), JCyL (VA28/99), RTN program of the European Union NANOPHASE (Contract No. HPRN-CT-2000-00167).

\section{APPENDIX: A SIMPLE CASE OF COMPARISON BETWEEN EXPERIMENTS AND THEORY FOR IMFP}

In core-electron photoemission experiments, an x ray excites core electrons of the material. These excited electrons jump up to the conduction band, leave the solid, and finally are collected in a detector. Briefly, the measured electron distribution consist of two main peaks due to the distinct core-electron binding energies of the surface and bulk atoms.

In order to resolve bulk from surface contribution we need to rely on a suitable fitting of each peak (usually with two gaussians of the same width). Once the collected signal is decoupled, it is possible to define the attenuation length $\lambda$, by the fraction of the total of signal that belongs to the bulk. The so-called relative intensity, $f_{B}$, assuming an exponential decaying law of the signal, is the given by

$$
f_{B}(E)=\frac{I_{\text {Bulk }}}{I_{\text {Total }}} .
$$

If now we assume that the contribution of the surface is only concentrated in the outermost layer (denoted by $j=0$ ), the relative intensity can be written as:

$$
\begin{gathered}
I_{\text {Bulk }}=\sum_{j=1} e^{-j d / \lambda(E),} \\
I_{\text {Total }}=\sum_{j=0} e^{-j d / \lambda(E),} \\
f_{B}(E)=e^{-d / \lambda(E)},
\end{gathered}
$$

where the sum runs over all the layers in the system. This is the standard experimental procedure to provide $\lambda$. We would like to remark that this technique assumes that the surface is concentrated on the first layer, and the widths of the surface and bulk atomic energies coincide. Without going into details of the reliability of such assumptions the important fact here is that experiment and theory do not measure (calculate) identical things. In a photoemission experiment, the key pa- rameter is the intensity. It is tautologic, but the experiment measures the photoelectrons that arrive at the detector, and this overweights photoelectrons with large mean free paths. In this sense, any attempt to approach more to experiment nature would mean to work with intensities, or treat the MFP calculation as a surface problem instead of a bulk one as done in our calculation. In this way let us briefly sketch the real nature of what is actually measured in a photoemission experiment. Experimentally to extract the IMFP, as showed before, the key quantity is the relative intensity, that can be written as sum of the probability for exciting the core electron on each layer:

$$
e^{-d / \lambda(E)}=\frac{\sum_{j=1} \sum_{n} \int d k A(k, n) e^{-d_{j} / \lambda(k, n)} \delta(E-E(k, n))}{\sum_{j=0} \sum_{n} \int d k A(k, n) e^{-d_{j} / \lambda(k, n)} \delta(E-E(k, n))},
$$

where $d$ is the interatomic distance, $d_{j}=j d$, and $A(k, n)$ the probability of exciting the core photoelectron to the Bloch state $(k, n)$. In a first approximation we can assume $A(k, n)$ to depend only on the energy.

Now if the $\{\lambda(k, n)\}$ are much larger than the interatomic distance the above, the sum in $j$ becomes straightforward, and the above equation can be simplified to

$$
\begin{aligned}
1-\frac{d}{\lambda(E)}= & \frac{\sum_{n} \int d k \frac{\lambda(k, n)}{d} e^{-d / \lambda(k, n)} \delta(E-E(k, n))}{\sum_{n} \int d k \frac{\lambda(k, n)}{d} \delta(E-E(k, n))} \simeq 1 \\
& -\frac{\sum_{n} \int d k \delta(E-E(k, n))}{\sum_{n} \int d k \frac{\lambda(k, n)}{d} \delta(E-E(k, n))}=1 \\
& -\frac{d}{\langle\lambda\rangle(E)},
\end{aligned}
$$

where by $\langle\lambda\rangle(E)$ we denote the average followed in Sec. III B. Hence, within this simple model we can see that only when the IMFP is much larger than the interlayer spacing the experimental IMFP and the theoretical one will describe same things. Obviously this model is too simplistic to give quantitative results, but illustrates that further theoretical and experimental work would bring necessarily light in this issue.
${ }^{1}$ J.J. Quinn, Phys. Rev. 126, 1453 (1962).

${ }^{2}$ J. Bokor, Science 246, 1130 (1989); R. Haight, Surf. Sci. Rep. 21, 275 (1995).

${ }^{3}$ Angle-Resolved Photoemission, edited by S.D. Kevan (Elsevier, Amsterdam, 1992).

${ }^{4}$ Y. Uehara, T. Fujita, and S. Ushioda, Phys. Rev. Lett. 83, 2445
(1999).

${ }^{5}$ P. Hohemberg and W. Kohn, Phys. Rev. 136, B864 (1964).

${ }^{6}$ L. Hedin, Phys. Rev. 139, A796 (1965); L. Hedin and S. Lundqvist, Solid State Phys. 23, 1 (1969); F. Aryasetiawan and O. Gunnarsson, Rep. Prog. Phys. 61, 237 (1998).

${ }^{7}$ I. Campillo, J.M. Pitarke, A. Rubio, E. Zarate, and P.M. Ech- 
enique, Phys. Rev. Lett. 83, 2230 (1999); I. Campillo, V.M. Silkin, J.M. Pitarke, E.V. Chulkov, A. Rubio, and P.M. Echenique, Phys. Rev. B 61, 13484 (2000); I. Campillo, J.M. Pitarke, A. Rubio, and P.M. Echenique, ibid. 62, 1500 (2000); I. Campillo, A. Rubio, J.M. Pitarke, A. Goldmann, and P.M. Echenique, Phys. Rev. Lett. 85, 3241 (2000)

${ }^{8}$ W. Kohn and L. Sham, Phys. Rev. 140, A1133 (1965).

${ }^{9}$ V. Silkin, E. Chulkov, and P. M. Echenique (unpublished).

${ }^{10}$ N.V. Smith et al., Surf. Sci. Lett. 282, 359 (1993).

${ }^{11}$ A. Fleszar and W. Hanke, Phys. Rev. B 62, 2466 (2000)

${ }^{12}$ M.L. Cohen, Solid State Commun. 92, 45 (1994); Phys. Scr., T 1, 4 (1982); W.E. Pickett, Comput. Phys. Rep. 9, 115 (1989); M.C. Payne, M.P. Tter, D.C. Allan, T.A. Arias, and J.D. Joannopoulos, Rev. Mod. Phys. 64, 1045 (1992).

${ }^{13}$ N. Troullier and J.L. Martins, Phys. Rev. B 43, 1993 (1991).

${ }^{14}$ We used a plane-wave band set with a 20 Ry energy cuttoff and a $20 \times 20 \times 20$ Monkhorst-Pach sampling of the Brioullin zone, needed to get an accurate description of the Fermi surface, fundamental for the lifetimes of electrons close to $\mathrm{E}_{f}$.

${ }^{15}$ P.M. Echenique, J.M. Pitarke, E.V. Chulkov, and A. Rubio, Chem. Phys. 251, 1 (2000).

${ }^{16}$ J. Lindhard, K. Dan. Vidensk. Selsk. Mat. Fys. Medd. 28, 8 (1954).

${ }^{17}$ The calculation of the RPA dielectric function has been done including properly local-field corrections and a large set of unoccupied states (up to an energy of $200 \mathrm{eV}$ above $E_{F}$ ).

${ }^{18}$ In practice, the $\delta$ function has been replaced by a Gaussian function.
${ }^{19}$ This has been checked using in Eq. (5) FEG matrix elements.

${ }^{20}$ D.W. Mermin, Phys. Rev. B 1, 2362 (1970).

${ }^{21}$ L.I. Johansson and B.E. Sernelius, Phys. Rev. B 50, 16817 (1994).

${ }^{22}$ It is also possible using another average to calculate the IMFP, $\lambda(E)=v(E) \tau(E)$, with

$$
v(E)=\frac{\sum_{n} \int d \mathbf{k} v(\mathbf{k}, n) \delta(E-E(\mathbf{k}, n))}{\sum_{n} \int d \mathbf{k} \delta(E-E(\mathbf{k}, n))}
$$

and $\tau(E)$ defined as in Sec. III A. In the studied range of energy and for these particular elements, $\mathrm{Na}$ and $\mathrm{K}$, both averages give the same results, although in other systems probably they would yield different results.

${ }^{23}$ At the energies where the FEG fits better than the group velocity, Fleszar's prescription $\left[v=\left(2<E_{k i n}>\right)^{1 / 2}\right]$ and the FEG velocity are almost identical (less than $5 \%$ of difference). This is the reason why we have not included the definition of the velocity given by Fleszar into our discussion.

${ }^{24}$ T.-W. Pi et al., J. Electron Spectrosc. Relat. Phenom. 107, 163 (2000).

${ }^{25}$ The experimental resolution $(\sim 0.1 \mathrm{eV})$ is also a mechanism that masks the crystal structure.

${ }^{26}$ J.B. Pendry, Low Energy Electron Diffrection (Academic Press, New York, 1974).

${ }^{27}$ D.W. Jepsen, F.J. Himpsel, and D.E. Eastman, Phys. Rev. B 41, 8075 (1990). 